\title{
Apendicitis y quiste torcido del ovario em una embarazada de cinco meses
}

pelos Drs. Manoel Rodrigues Lopes $e$ Juan Malet, da 1.a Clinica Obstateica da Faculdade de Medicina de Montevideo.

\section{I - DISCRIPCION CLINICA}

Dar publicación a una observación clinica, por modesta que ella sea, cual tudo la lleva solamente el fin de llamar la aiención sobre hechos que pueden servir luogo para despistar en clinica o pensar en complicaciones análo gas, es obra siempre de indicutible valor. Es ésta el fin que nos lleva a publicar nuestros trabajos sobre estes cuadros agudos que, asociados, vienen a complicar el embarazo en el curso del quinto mes de la enferma considerada. Las publicaciones que se han hecho sobre estos separadamente, si bien no son muy frecuentes, no dejan sin embargos de presentarse en la clinica. Sobre este mismo tópico se han publirado y se han expuesto perfectamente casos similares, per lo que no tiene más pretension esta anotación clinica que contribuir a enriquecer más las observaciones en este capitulo tan interessante de la ginecologia.

Los cuadros apendiculares, complicando o apareciendo en el curso de la gestación, son vistos con relativa frecuencia. y hoy la literatura médica está enriquecida con observaciones que han dado lugar a trabajos altalamente interessantes. Lo mismo podemos decir de los quistos torcidos de ovarios, que a pensar de su menor 
frequencia no por eso dejan de verse, complicando a veces la gravidez. Lo que tiene de interesante nuestro caso clinico es la asociación de estos dos cuadros agudos de vientre: apendicitis aguda, por un lado, $\mathrm{y}$ torsión de un quiste ovárico derecho; sorprendente asociación, que llama la atención por lo interesante del caso $\mathrm{y}$ por la impossibilitad de llegar a un diagnostico clinico. puesto que la semiologia clinica del uno enmascara completamente la semiologia clinica del otro. Tal fué nuestro caso, en que segun veremos en la descripción clinica del mismo, el diagnostico fué completado en el acto operatorio.

OBSERVACION CLINICA - Dora L. de E. Ingresa a nuestra clinica el 8 de Febrero del año 1928 con 38,5 gráos de temperatura; pulso, 114; lengua seca y saburral.

INTERROGATORIO - Antecedentes hereditarios y personales carecen de importancia. Interrogada sobre su afección. nos dice que hace cinco meses le faltan sus menstruaciones, que siempre habian sido regulares. Hace cinco dias sintió un fuerte dolor en la fosa derecha, acompañado de vómitos y nauseas que todavia persisten.

SEMIOLOGIA - Por la inspección se nota una tumoración abdominal baja, a convexidad superior y realzada en este caso debido a la delgadez de las paredes abdominales. Inmovilidad respiratoria abdominal completa.

Por la palpación se sacan pocos datos, debido a la exagerada contractura de defensa de la pared abdominal. Vientre muy doloroso espontaneamente, con un maximun de dolor en la fosa iliáca derecha, donde la presión encuentra un punto de exquisito dolor, cuya localización corresponde al Mac Burney. Dolor que aparece también a la decompresión.

Por el tacto se encuentra cuello reblandecido, utero aumentado pero impossible de limitar completamente a la palpación; debido a la defensa abdominal; fondo de saco derecho doloroso y al parecer ocupado. 
Por el conjunto de datos clinicos que recogimos del examen semiológico y junto a los antecedentes de debut brusco, estado ebril, dolor exquisito en la fosa iliaca derecha (Mac Burney), constipación, vómitos, etc., dan a este cuadro agudo de vientre la fisonomia clinica de un sindroma apendicular, desprendiéndose de éste el diagnostico de apendicitis aguda.

Ordenamos bolsa de hielo, toniîicación y una leucocitosis; mientras inyetamos suoro fisiológico. La leucocitosis, cuyo resultado nos es dado en seguida, da: 22.800 glóbulos blancos por milimetro cubico. Es en estas condiciones que, al hacerme cargo de la guardia como médico interno, veo a la enferma, ordenando la la operación, después de haber examinado y constatado les mismos sintomas y haciendo el mismo diagnóstico clinico: - apendicitis aguda.

INTERVENCION OPRATORIA - Anestesia general al éter. Ayudado por la Dra. Ema E. Tiribocchi, hago una incisión de Mac Bunrney; abiertos los distintos planos he incidido el peritoneo; caemos a la cavidad abdominal. donde por la simple inspección constatamos una masa redonđeada equimólica con la cacacteristica de los quistes de ovario torcidos, con hemorragia intraquistica. Agrandamos un poco la incisión, lo suficiente para dar paso a la mano, y comprobamos que se trata de un quiste de ovario del tamaño de una cabeza de adulto, completamente libre, sin adherencias. En vista de lo cual resolvemos extirpalo, para lo cual con un trocar lo puncionamos, y luego, después de estar flácido debido a su parcial vaciamiento, lo exteriorizamos, ligando su pediculo (el cual tenia tres vueltas de torsión), extirpándolo.

Explorando nuevamente la fosa iliaca derecha, asiento central de la sintomatologia, nos encontramos con un apéndice duro, turgente e intensamente congestionado, indice de un proceso apendicular agudo, que daba la completa comprobación a nuestro diagnostico. Lo extirpa- 
mos. luego de lo cual, observando nuevamente el vientre. comprobamos un utero grávido de cinco meses. Estando las cosas en perfecto estado, cerramos el vientre en tres planos, teniendo la enferma un excelente post-operatorio, dándosele de alta a los nueve dias, continuando normalmente su embarazo.

En resumen, tenemos que el acto operatorio agrega a nuestro diagnóstico clinico de apendicitis aguda, el de quiste torcido del ovario; de maneira que el diagnostico es de: apendicitis aguda y quiste torcido de ovario en una embarazada de cinco meses.

CONSIDERACIONES Y REFLEXIONES QUE NOS SUGIERE EL PRESENTE CASO - Es indudable que nuestra observación tiene un interés clinico de alto valor debido a esta rara asociación de dos procesos agudos de de vientre que se superponen, ocultandose el uno tras la sintomatologia del otro. Frente a un caso similar, en que la semejanza del cuadro clinico de los dos procesos hace impossible el diagnostico diferencial de ambos y el de su superposición, en que la contractura de la pared abdominal y del contenidon del utero grávido de cinco meses en la cavidad abdominal hacen impossible el diagnóstico clinico exacto, - el clinico hace y hará siempre el diagnóstico de apendicitis, siendo impossible estabelecer la concomitancia con el de quiste torcido de ovario, pues - vuelvo a repetirlo - la sintomatologia fisica lo mismo que la funcional, se enmascara completamente, según lo hemos visto en los dates que hemos expuesto.

Otro punto interessante e impossible de diagnosticar con certeza, siendo hipotéticas todas las conclusiones que pudiéramos sacar al efecto, será a saber si estes cuadros agudos de vientre, que en nuestro caso se superpusieren, han tenido un comienzo simultáneo e, como es más factible presumir, han nacido uno con anterioridad al otro, y a cual, en este último caso, le correspond la 
prioridad en la sintomatologia que caracterizó nuestra observación, y que independientemente cada uno de elles podia por si solo haberla dado por separado. Agregamos a este dificultad todavia el haber visto a la enferma un largo tiempo después de haber hecho su aparición el cuadro agudo.

Es indudable que si hubiéramos tomado a la enferma en el comienzo de su afección, cuando la pared abdominal no hubiera estado contracturada, nos hubiera permitido, por el tacto vaginal combinado, la percepción clara del quiste ovárico, a pesar de que el cuerpo uterino grákido, interpuesto entre los dedos exploradores, hubiera dificultado en parte también este diagnóstico.

Interesante también resulta, como digno de anotarse y hacer resaltar en el acto operatorio, es que: una vez hecho el diagnostico y verificada la liberdad del quiste, la punción, evacuando parcialmente su contenido, nos facilitó su exteriorización, per lo que, junto a su localización derecha, fué fácil extirparlo por la misma incisión apendicular, evitando la malaxación uterina, que hubiera traido la interrupción del embarazo, provocado el aborto. Interesante resulta, pues, $\mathrm{y}$ es justo hacerlo remarcar, que por la incisón de Mac-Burney, de unos ocho centimetros, cuyo fin clinico era hacer la extirpación del apéndice en el acto operatorio, nos fué posible extirpar, por la feliz coincidencia de la localización derecha de la formacion tumoral, un quiste torcido de ovario del tamaño de una cabeza de adulto, seguido luego por apendicetomia com conservación del embarazo y com la continuación clinica de un magnifico post-operatorio.

Passamos ahora al estudio y descripción anátomopatológica del apéndice extirpado.

\section{II - DESCRIPCION ANATOMO-PATOLOGICA}

Dividiremos la descripcion anatomo-patológica correspondiente a nuestro apéndice ya extirpado en: a) Macroscópica, y b) Micróscopica. 
a) Examen macroscópico: EI apendice media unos 11 ctms., presentando un diametro algo mayor que le normal. A la observación mostraba ser un apéndice congestivo; al tacto, de consitencia dura, y al corte dada en ciertas zonas la impresion de haber tejido esclerosado. Encontramos un cálculo obstruyendo la luz del apéndice, en la mitad del mismo. La mucosa estaba congestionada, presentado en ciertas perciones manchas hemorrágicas.

b) Observaicón microscopica: 1. Mucosa: a) Epitelio: Observado a pequeño aumento aparecen segmentos de la mucosa en que el epitelio está destruido; a gran aumento se constata: hipersereción, correspondiente a las celulas epiteliaes; hipertrophia de las glandulas de Lieberkühn, rodeadas por un proceso inflamatorio donde se observa numerasas células linfocitarias y algunos monucleares.

b) Basal: Normal.

c) Corion: Se observan los corpusculos linfoideos hipertroficos y capilares congestionados. Existe asociación corpuscular anormal, observandose además en las porciones de mucosa intercorpuscular, un processo de infiltración, y en parte de edema ligero, con abigarramiento celular a gran predominancia linfocitaria. Se observan también, y en numero crecido, cólulas a grueso nucleo, nucléo jóven, que se tiñe intensamente por los colorantes nucleares y rodeados por un halo de protoplasma rosado. Las hay de formas ya redondeadas, ya alargadas. Son nucleos y células conjutivas, que acompañan al proceso inflamatorio. Observamos también macrófagos, como circunscribendo los corpusculos linfocitarios, asim como también leucocitos, los más neutrófilos.

En la microfotagrafia (Fig. No 1, Microfotogr.) se observa: Fibrillas conjuntivas; Células embrionarias, y Linfocitos numerosos.

En ciertas porciones de la mucosa se observan glóbulos rojos, pigmentos sanguineos y algunos elementos representativos de la serie blanca. 
Hicimos un esquema, tal como se ve (en la Fig. No5 dibujo), compuesto de la observación de varios campos de la mucosa, en la cual observamos (A) Monucleares; (B) Un vaso con paredes proliferantes; (C) Elementos inflamatorios de la pared vascular; (D) Linfocitos numerosos; (E) Leucocitos y en (F) vemos un capilar en el quense ve su luz ocupada por glóbulos rojos y restos de nucleos celulares.

Hemos observado también en ciertas zonas de la mucosa una mayor cantidad de nucleos y restos nucleares fagocitados, como asimismo mayor numero de leucocitos. Pudimos observar también, tal como se ve (en la Fig. No 2, Microfotogr.), haces cológenos espesos, haces conjuntivos delgados, y leucocitos con nitidos, empleando el método de Rio Hortega, Tecnica general, variante a).

Passemos a estudiar ahora la segunda capa, la musculosa. Empleamos la Hematoxilina Eosina, la Hematoxilina férrica, picrofuchsina, Rio Hortega etc. En la (Fig. No 3, Microfotogr.) pudimos observar capilares congestionados en los espacios intermiofibrilares e extrafibrit lares, como asimismo células linfocitarias formando agrupaciones inflamatorias entre los haces musculares. Hemos observado también en esta capa edema y algunos haces disociados. Menor cantidad de leucocitos y pocos monucleares.

Pasemos a estudiar, por ultimo, la subserosa y serosa.

Vemos en la (Fig. No 4, Microfotogr.), leucocitos y linfocitos, estando estos en mayor numero que normalmente. Algunos de ellos están agrupados sobre los capilares y otros esparcidos en el estromas conjunctival. Se observa también - lo que si empleando mayor aumento - leucocitos de diversas formas, a nucleo fragmentado, células fagocitarias. Restos nucleares. Se observa también numerosos mononucleares, algunos de ellos con un protoplasma debilmente teñido; en otros se observa el 
nucléo solamente. Hay tambien células a largos prolongamientos, finos y ramificados, ocupando ora los lugares no muy irritados ya alredor de los vasos, tomando diversa forma ya en nucleo, ya en sus prolongamientosi Estas células son para nosostros células emigrantes inflamatorias, estudiadas con diversos métodos de tinción y descriptas con precisión por diversos autores.

Aqui también quisimos completar la descripción por medio de un esquema que resumiese la vision de diversos campos. Y observamos (Fig. No 6 dibujo) lo siguiente: (A) Son células linfocitarias, que aqui (lo mismo que en Fig. No 4, Microfotogr.) se agrupaban como formando islotes linfocitarios (B) Encontramos también alredor de los vasos fibroblastos. (C) Se observan también mononucleares. (D) Se ven leucocitos, con diversa forma en su nucleo, bi o trilobado. (E) Mononucleares y celulas embrionarias, junto a una magna de sangre coagulada; dentro de este vaso se ven restos nucleares ' $y$ un proceso de perivascularitis manifiesto. Y por ultimo, (G), son los nucléos del endotelio inflamado del vaso considerado.

\section{RESUMEN Y CONCLUSIOES CLINICAS Y} ANATOMO PATOLOGICAS.

Conclusiones clinicas - Como vemos por el caso que exponemos frente a un cuadro de fosa iliaca derecha pensamos en una apendicitis, diagnostico que se imponia por los resultados del examen, pero datando el cuadro de varios dias, la defensa de la pared hace que sea imposible el diagnosticar quiste torcido del ovario. Siendo un encuentro de operación la asociación apendiculo ovárica aguda cuando el cuadro no es reciente. La intervención en nuestro caso fué hecha con el diagnostico de apendicitis aguda resolviendose el acto operatorio con una incisión de Mac Burney por la que fué posible extirpar facilmente el quiste debido a que este no era adherente. 
B) Conclusiones anatomo patólogicas. - 10) Macroscópicamente el apendice observado sus dimensiones algo mayor que la normal. Al tacto impressiona en ciertas porciones como tejido escleroso. Encuentrase en la mucosa hemorragias puntiformes y un calculo obstruyendo la luz del apéndice; 2o) Microscópicamente: observamos (A) en la mucosa: Un proceso inflamatorio crónico agudizado - confluencia de los fóliculos, destrucción del epitelio de revestimiento, hipertrofia grandular, y focos hemorragicos. B) En la musculosa: encontramos infiltración linfocitaria y lijero edema. (C) en la serosa: gran cantidad de linfocitos y algunos leucocitos y células reaccionales emigrantes.

Luego concluimos del examen del apéndice considerado: "Trátase de una apendicitis crónica que sufrió un nuevo empuje inflamatorio agudo".

\section{BIBLIOGRAPHIA}

1 Aschoff - Die Wurmforsatzenzün dung, Iena 1908.

2 " - Deutsche patolog., Gellsch 1904 - 1907.

3 " - Medizin Klinik - 1905.

4 Dieulafoy - Bull de l'Acad. de Medecine, Paris 1906.

5 Flesch Münch - Med. Wochensch - 1907.

6 Fraenüel - Deutsche Med. Wochensch, 1904.

7 Ghon e Nambu - Ziegler's Beito f Path. Anat. Vol. LIII, 1912.

8 Hausemann, Sonnemberg e Krauss - Discussione sulla patogenesi dell appendicite (Kongress f. inn Medizin 1906.

9 Kretz - Verhandl d. Deutsche pathol. Gessellsch 1906 - 1910 .

10 Kretz - Mitteil aus de Prenzgeb, etc. - 1917.

11 Laewene Reinhardt - Nünch Mediz. Wochensch N.o $50-1920$.

12 Kretz - Zeitsch f. Hig. Vol. XXVIIl - 1907. 
13 Oberndorfer - Ergbnisse d. Alleg Path., Vol. XXIII - 1909.

14 Oberndorfer - Franüf. Zeitschr f. Patholg. Vol. Il - 1918.

15 Oberndorfer - Mediz. Klinik - 1911.

16 Rem. Frankf. - Zeitsch f. Patholog., Vol. XIX - 1917.

17 Rubesch e Sugi. - Beitrage Z Klin. Chir. - 1912.

18 Sonnenburg - Deutsche Med. Wochensch N.o 13 - 1912.

19 Sugi - Virchow's Archiv. Vol. CCX - 1912.

20 Watzold - Zeigler Beitr f. Pat. Anat. Vol. XLIII - 1907

21 Cagnetto - Virchow's Archiv. Vol. CXCVIIl - 1909.

22 Hausemann - Verhandl d. Deutsche Patholog. Gesellsch - 1914.

23 reckKe Münch Med. Wochensch - 1913.

24 Oberndorfer - Deutsche Mediz. Wochensch - 1906.

25 Oppenheim Frankf - Zeitsch f. Patholog. - 1909.

26 Sissojeff - Virchow's Archiv. Vol. CCV - 1911.

27 M. M. Rougemont et M. Dechaume - Presse Medicale 1927 pag. 273 - Torsion d'un quiste de l'ovaire droit et appendicitis.

28 Cazin - Appendicitis et inondation peritoneal, consecutive a un rupture de Kyste ovarique tordu $\operatorname{Arch}_{i} v$ d'obstetri $_{i}$ que - 1912.

29 Vauchert V. - Appendicitis Salpingoovaritus droite - Gaz. Med. de Picardie - 1897.

30 Treub. H. - Appendicilis et Parametritis - Revue de Gynecologie et de chirurgie abdominale - Pag. 263.

31 La torsión simultánea del apéndice y quistes del ovario derecho - (Contribuciones anatómicas y clInicas: Dr. Clivio Nario.

N. da R. - Foi prejudicada a reproducção de quatro microphotographias que acompanhavam este trabalho. 